\title{
Centroids of finite dimensional associative dialgebras
}

\begin{abstract}
We introduce the concept of centroid for associative dialgebras and study its some properties. An algorithm to find centroids of algebras is given. We apply the algorithm to determine the centroids of lowdimensional dialgebras.
\end{abstract}

Keyword: Associative dialgebra; Centroid; Derivation; Central derivation 